\title{
Mark Reynolds $\mid$ A Comparative Geometric Analysis of the Heights and Bases of the Great Pyramid of Khufu and the Pyramid of the Sun at Teotihuacan
}

Mark Reynolds examines the Pyramid of the Sun at Teotihuacan and the Great Pyramid of Khufu from the point of view of geometry, uncovering similarities between them and their relationships to the Golden Section and $\pi$.

\section{Introduction}

Looking back into the murky mysteries of ancient times, there are reminders of past glories in the art, architecture and design of our ancestors, and in the number systems they employed in those designs, expressed in the geometry they used. Among these works are the mammoth pyramids that dot the Earth's surface. Accurate in their placement as geodetic markers and mechanically sophisticated as astronomical observatories, these wonders of ancient science stand as reminders that our brethren of antiquity may well have known more than we think. In our attempts to understand and decode these objects of awe, we realize that the winds of time and the ignorant hands of humanity have eroded the precise measurements and canons that were infused into these monuments. By their sheer magnitude ${ }^{1}$, the pyramids tell us that their builders clearly wanted future civilizations to not only notice them, but to also investigate them in an attempt to find out what knowledge these masons and priests possessed regarding the world and the universe; although the precision of the structures may be missing, we can still see the intentions through the geometry that remains. This study was undertaken with that in mind.

In the measurement and geometric analysis of an ancient structure, it is often necessary to combine scientific and mathematical objectivity with an open mind that will permit an understanding of what appears to be the intentions of the maker...that is to say, educated common sense. Ideally, one would rejoice in having the original plans and documents that would clearly explain the precise measures, or to have an opportunity to measure the work as it was at its completion. More often, the problem can be reasonably solved by researching documents and texts on the piece to be analyzed, and by looking for reputable and reliable scholars that have gone before to do similar studies. By combining this approach with drawings and photographs and, where possible, with measurements in situ, a sensible study can be undertaken. 
Scholars and writers have presented studies, surveys and mathematical information to support their beliefs that the two pyramids under discussion incorporate mathematical formulae that refer to $\pi$, the size of the earth, the squaring of the circle and other numerical and esoteric data. Some of the information is valid, some conjecture, while other items in print must be viewed at least skeptically, if not completely ignored. Of course, there is always the difficulty presented by the mathematical certitude that is perpetually in conflict with the intuitive nature of the artist. Mathematicians and artists will disagree on whether a line has thickness, and scholars will argue over the ability of a geometer to rectify the square or to be completely accurate with an irrational number. ${ }^{2}$ Such are the perils of anyone stepping into the arena of $\pi, \varphi, \theta$, the root rectangle system and other incommensurables. At the risk of incurring arguments over these issues, let us continue, with the hope that the mathematician and the artist will reach a common ground of mutual respect and agreement in their shared appreciation for the beauty involved.

\section{Dimensions of the Great Pyramid and the Pyramid of the Sun}

My initial investigation was focused on a verification of the base/height measures of the Great Pyramid of Khufu (Greek: Cheops) at Gizeh, Egypt, and the Pyramid of the Sun at Teotihuacan, Mexico, and the precision of each with regard to $\pi$ to determine if one was more accurate than the other. I was quite content to use rational approximations for $\pi$, such as the Egyptian value of $256 / 81$ or the 22/7 of Archimedes of Syracuse, one of my heroes. (Archimedes was murdered by Roman Soldiers for doing geometric constructions in the dirt.) I find this approach more helpful in appreciating the flavor of the works, and the intentions of the master masons who were involved in constructing them. However, a calculator is indispensable for calculating percentatage deviations. Below is a chart that will be used in the presentation of the comparative analyses of the two pyramids. It contains the measures I used in my study of the two pyramids. ${ }^{4}$

Table 1.

\begin{tabular}{lll} 
& Great Pyramid of Khufu & Pyramid of the Sun \\
\hline Height & $481.3949 \ldots$. feet & $233.5 \ldots$. feet \\
\hline Base Perimeter & $3023.16 \ldots$ feet & $2932.8 \ldots$ feet \\
\hline Side & $755.79 \ldots$ feet & $733.2 \ldots$ feet \\
\hline 1/2 Side & $377.895 \ldots$ feet & $366.6 \ldots$ feet \\
\hline Angle of slope & $51.827 \ldots$ degrees & $32.494 .$. degrees
\end{tabular}

The rectangles generated from the height of each pyramid and its half-base, when the halfbase is considered to be 1 :

Great Pyramid 1.2738853 ... : 1 (deviates by $0.15 \%$ from the 1.2720196 value for $\sqrt{ } \varphi)$. 
Pyramid of the Sun $.6369339 \ldots$ : 1 (deviates by $3.06 \%$ from the 0.618034

value for the reciprocal of $\varphi$ ).

The ratio of the base perimeter to the height:

Great Pyramid 6.2800001... : 1 (deviates by $0.05 \%$ from the 6.2831853 value for $2 \times \pi)$.

Pyramid of the Sun $12.560171 \ldots$ : 1 (deviates by $0.05 \%$ from the 12.566371 value for $4 \mathrm{x} \pi$ ).

The ratios of the bases to one another:

$$
\begin{aligned}
& \text { Great Pyramid/Pyramid of the Sun } \\
& \text { Pyramid of the Sun/Great Pyramid }
\end{aligned}
$$

As stated by Graham Hancock and others, the two pyramids are "almost" or "very nearly equal" to one another in base perimeter. (Along with this thought there is also the statement that the Pyramid of the Sun is "almost" half the height of the Great Pyramid; this will be addressed later.) Because of the closeness, there is often the tendency to state that they are equal. One of the major points of the paper is that there is a difference, an ever-so-slight one that can be explained and demonstrated by the geometric constructions presented here. The Great Pyramid is 1.03... times larger than the base of the Pyramid of the Sun. Conversely, the base of the Pyramid of the Sun is $97 . . \% \%$ of the Great Pyramid's base. Is this an accidental similarity, or a telling coincidence? Were the two monuments built by totally separate cultures, or was there a shared cultural code?

As a geometer, my curiosity was piqued by these questions and issues, and I wanted to proceed to geometric constructions to investigate the relationships, especially because the structures under discussion were made by cultures who revered geometry as a gift from the gods. Our brethren of ancient architecture probably did not have advanced calculating technologies, and it may be possible that they were not so concerned with incommensurable, irrational and transcendental numbers, for in the geometry, these qualities exist by construction, not measurement. Measure was used primarily for calculation of heavenly and earthly phenomena. However, they were keenly aware of circles, cycles and spheres, for these things were visually observed. What is evident now is the fact that they were concerned about relationships surrounding the concepts of unity, duality and multiplicity, of how all could be melded into ONE. Schwaller de Lubicz writes in The Temple of Man that for the Egyptians, ONE is the largest number, and all other numbers are fractions of this primal and universal unity. ${ }^{5}$ The real issue isn't how accurately the priestly geometers could calculate and use $\pi$ and $\varphi$, but rather, how they could integrate the two into one cohesive unity. The greatest difficulty with this coupling is that of the "square peg in a round hole", or in geometer's terms, the classical problem of quadrature, squaring the circle. There are significant relationships between the circle and the square, but there is a fundamental difference between them, not only geometrically or 
numerically, but symbolically and philosophically as well. The circles and spheres in the dome of the sky are contrasted by the four directions of the Earth and its four seasons. It is difficult to find squares above and circles below. The ancient master builders sought the marriage or union of these opposites in the temple; to them, this was the extension of spirit into matter.

Through all of this, the common thread was the search for the geometry that tied $\pi$ and $\varphi$ together. This union can be consummated with esoteric relationships:

- 4 divided by $\sqrt{ } \varphi$ is approximately equal to $\pi(4 / 1.2720196=3.1446055$, a percentage deviation of less than $0.01 \%$ ).

- $5 / 6$ of $\varphi$ squared is approximately equal to $\pi(6 / 5 \times 2.618034=3.1416408$, a percentage deviation of less than $0.002 \%$ ).

- $\varphi$-squared plus 1 , divided by $\varphi$ is approximately equal to $2([1.618034+1]$ $/ 1.618034=2.236068$, a percentage deviation of $11.8 \%$ ).

Arguably, there is no true equality in these figures because we are dealing with roots, irrationals and transcendentals. The importance lay in the attempt to make their creations as perfect as possible with what was there. The question is only one of localities or global communities: if the Egyptians reckoned this way, did their counterparts on the other side of the world independently and coincidentally reckon things in the same way? Or, were the cultures building pyramids a shared group of masons traveling the globe, doing similar things in greatly differing places? There may not be a definitive answer, but I suggest that there is a unique and specific geometric construction that uses the circle and the square, and $\pi, \varphi$, and $\sqrt{ } \varphi$ in an elegant and mysterious way, and it is far too subtle to be an accident. It is a construction that is used to raise the elevation of the Great Pyramid that is based on the golden section, a construction widely known to us today. There is, however, a curious and minute measure that is also generated, a small function generated from the original, that yields the unique relationship with the Great Pyramid and the Pyramid of the Sun. What is most intriguing is that the construction involves the more mysterious $\sqrt{ } \varphi$, and not its more well known relative, $\varphi$. But let us now turn our attention to these constructions that connect the Great Pyramid to the Pyramid of the Sun.

$\varphi$ and root- $\varphi$. Let us begin with $\varphi$, the golden section ratio (Figure 1). This ratio is the division of a line segment or a magnitude into what Euclid termed "mean and extreme ratio", that is, a division such that the lesser part is to the greater as the greater is to the total. ${ }^{6}$ It is also found in the pentagram and pentagon as the ratio between a side and a chord; if the side is equal to 1, the ratio is equal to 1.6180339: 1.7 The "Divine Proportion", as it is sometimes called, can be found throughout nature: in the bones of the fingers, hand, and forearm, the location of the navel and where the fingertips touch the thigh in relation to the height of the adult human body; in the spiral seed patterns in a sunflower or daisy; in a spiral nebula light years from the Earth; in the curvature of certain seashells. Geometrically, the spiral nebula and the nautilus shell differ only in size, because each exhibits a logarithmic spiral based on the same golden section and $\sqrt{ } \varphi$ numbers. The golden section is one of the more well-known ratios that have been used throughout history in the making of things calling for certain types of harmony and proportion. The golden section ratio can be constructed in several ways. The half-diagonal of the square and the diagonal of the double square can each be used. In Figure 
$2, \mathrm{~KB}$ can be a segment of any length that we will call 1 . By constructing a double square AKBL on this length, the golden section of it can be found at G. The specific steps are:

1. Draw any length KB.

2. Divide the length KB in half. (Use a measure, a bisector, or the Vesica Piscis.)

1. These two lines, $\mathrm{KO}$ and $\mathrm{OB}$, are the short sides of a double square $(\sqrt{ } 4)$.

4. Construct the double square.

5. Draw a diagonal KL.

6. With compasses

7. Taking the compasses, put the pin in $\mathrm{L}$, and open to $\mathrm{B}$, drop LB onto the diagonal $\mathrm{KL}$ at $\mathrm{q}$.

8. With the pin in $\mathrm{K}$, open the compasses to $\mathrm{q}$, and rotate $\mathrm{Kq}$ to $\mathrm{KB}$ at $\mathrm{G}$.

$\mathrm{G}$ is a golden section of $\mathrm{KB}$, and $\mathrm{BG}: \mathrm{GK}:: \mathrm{GK}: \mathrm{KB}=1 / \varphi: 1:: 1: \varphi$. The simplest way is to generate the ratio is through its better-known relative, the golden section rectangle (Figure 3):

1. Draw square AKMZ.

2. Locate the midpoint of a side of the square $A K$ at $\mathrm{H}$.

3. The half-diagonal of the square, HM, rotated through the arc MR to the vertical position to become HR.

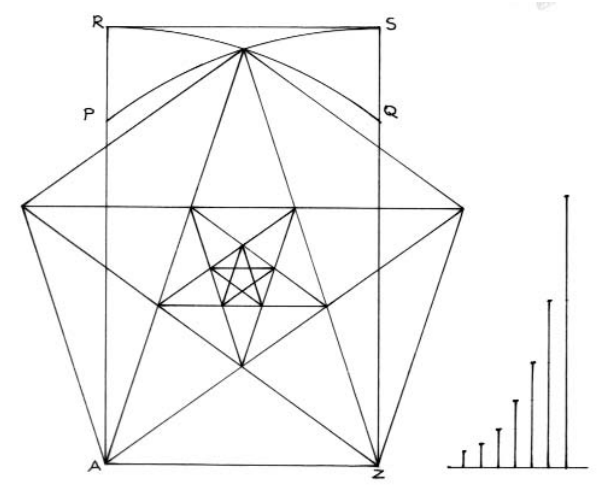

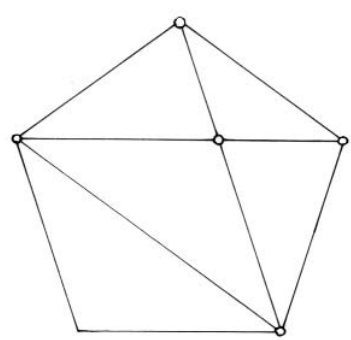

Fig. 1

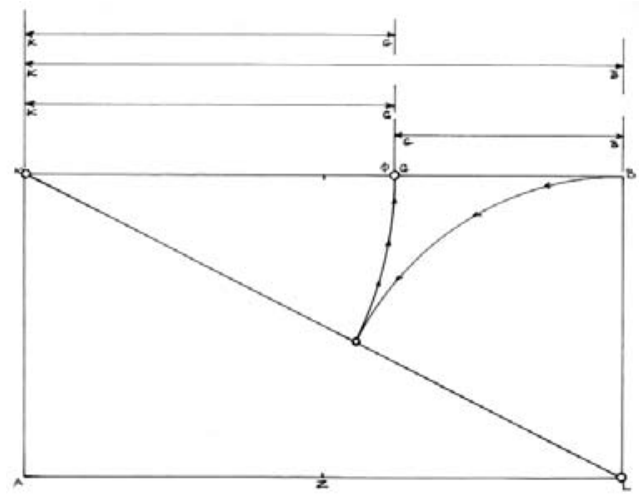

Fig. 2 


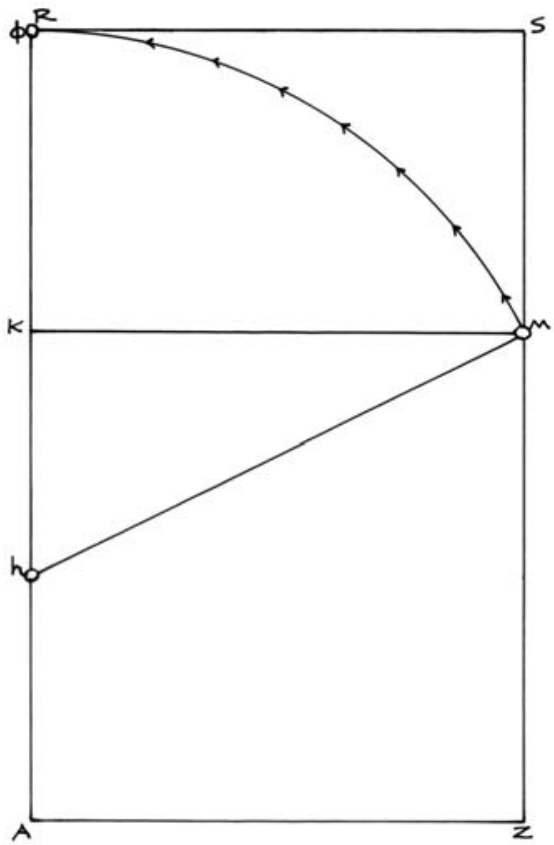

Fig.3

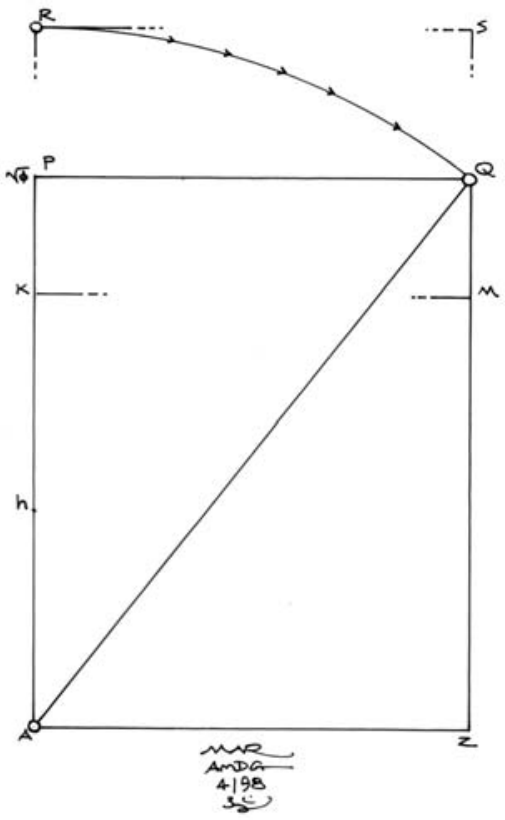

Fig. 4

Rectangle ARSZ is a golden section rectangle. Point $\mathrm{K}$ is also at a golden section of AR. This rectangle is composed of a square, AKMZ, and KRSM is also a golden section rectangle. The following relationships are present:

AZ:AR :: $1: \varphi$.

Now that the golden section rectangle has been generated, we will now proceed to draw a new rectangle called the square root of the golden section (Figure 4):

1. Draw golden section rectangle ARSZ.

2. It is next required that the long side of the rectangle ARKZ, that is, AR, be rotated through arc RQ to point $\mathrm{Q}$ on the opposite side, SZ, of the rectangle.

The triangle that is formed, AQZ, is sometimes referred to as the "Triangle of Price", after W. A. Price (who named it after himself!). This triangle is the only right, scalene triangle whose sides are in a geometric progression, a progression based on the square root of the golden section: $1, \sqrt{ } \varphi, \varphi(1,1.272 \ldots, 1.618 \ldots)$. Most notably, this triangle is, as we shall see, associated with quadrature (squaring the circle) and the relationship between the sizes of our Earth and moon. The simplicity of these constructions conceal the power and complexity that underlies the geometry.

The angle of slope for the GP Pyramid is very nearly $51.827291 \ldots$ degrees or $51^{\circ} 49^{\prime} 38^{\prime \prime}$. An interesting aside is the fact that the 7-sided figure, the heptagon, has an angle from its 
center that subtends an angle of $51^{\circ} 25^{\prime} 42^{\prime \prime}$, a mere $0^{\circ} 23^{\prime} 55.3^{\prime \prime}$ difference. Not perfect, but very, very close.

\section{Geometric Construction of the Pyramids}

Now that we are familiar with the bases, we can begin to construct the geometry of the pyramids. In this section, I will be giving procedures and explanations for the geometric constructions found in the paper. Rather than presenting cold and technical procedures, I would like to briefly make a few points on the philosophical aspects of the geometer's art. With these thoughts in mind, it is my hope that the constructions will take on added meaning.

The geometer believes that as the drawing is done, the geometry exerts a certain quality to its maker, similar to the gold in the alchemist's cucurbit. Geometric devices are powerful in and of themselves as well as for the purposes for which they are drawn. The geometer will usually begin a drawing from the circle, square, and/or the triangle, with the square being the most frequently used, and the most practical. As a general rule, philosophical geometry considers $\pi$ to be in the realm of the circle, $\varphi$, and in that of the square, roots and rectangles in that of the square as well, and polygons and regular solids in that of the circle/sphere and the square/cube.

The most important points to keep in mind when examining the drawings presented below are:

- All the drawings here emanate from and are generated by the square.

- The side of this square is always ONE (=Unity or wholeness); understanding this, and that all the other numbers that are discussed are in relation to this ONE, is the simplest way to comprehend the other measures, as they all rely on 1 as the basis for comparing their magnitudes and ratios.

- The square is the expression of Unity on the Earth, and that the circle is the same expression of Unity for that which is above and around.

- All rectangles are the expansion of the square.

- The orientation of the rectangle emphasizes either the horizon of the earth, or the vertical relationship between that which is below with that which is above.

- The geometric construction attempts to manifest a higher absolute system into the physical world; as such, it attempts to represent both rational and irrational magnitudes; in philosophical, or sacred, geometry, there are absolute figures (circle, square, triangle, cross, spiral) in a higher realm that become relative when manifested in the physical realm (variations occur, for example, when various line weights and media are used).

- In spite of the perfection of what I call "Ideal Numbers" (e.g., $(\sqrt{5}+1) / 2=\varphi$, or $1 / \varphi)$, coupled with the precision of the geometer's compasses, straightedge, and set squares, it is impossible to make a perfect drawing or building; thus the eternal duality of the absolute vs. the relative.

It is also important to note that, in the procedures for the geometric constructions used in the Pyramids, there is an underlying organic quality that functions in the drawings. First, the half-diagonal of the square $(\sqrt{5} / 2)$ is moved to generate the golden section. This is done by 
attaching the magnitude $1.118033 \ldots$ to one-half $(.5)$ the side of the square $(0.5+1$. $11803 \ldots=1.618033 \ldots .{ }^{8}$ Second, a very specific organic action is taken on a function that is in and of itself already organic. This step requires that the golden section length be moved from its upright 90 -degree angle to become the root of the new rectangle called the $\sqrt{ } \varphi$ rectangle, having the golden section symbolically growing as this root as represented by the diagonal of this new rectangle. It is the root that grows through this ratio. The underlying truth is that geometry appears to be a hard and mechanical device, but under the surface, there is an occult and earthy organic quality that is manifested either by the life force, or somehow through the geometry itself. It is in the nature of geometry itself to manifest growth, and $\varphi$ is its most organic function. Beginning with the Triangle of Price AQZ in Figure 4 and using bilateral symmetry, we can construct the Great Pyramid's elevation by doubling the base AZ at $\mathrm{Z}$ to be $\mathrm{AL}$ (do this by placing the compasses on $\mathrm{Z}$ to swing $\mathrm{AZ}$ to $\mathrm{AL}$ ). Next, draw the slope $\mathrm{QL}$ to complete the elevation (Figure 5). The resulting triangle, AQL, is now an isosceles triangle with sides $=1.618,2$, and 1.618, and the vertical axis (called cathetus) is = $1.2720196 \ldots$, or $\sqrt{ } \varphi$. (Points $\mathrm{p} 3$ and $\mathrm{M}$ in this figure will be discussed later.)

Figure 6 combines the triangular elevation of the Great Pyramid (AQN) with the plan of the square base (BKJL), seen together in one drawing. This is an important drawing because it plays a key role in later drawings of both pyramids, enabling us to see how both have been formed.

We will now construct the slightly smaller base of the Pyramid of the Sun. Starting with the drawing of the the combined elevation and plan of the Great Pyramid shown in Figure 6, use

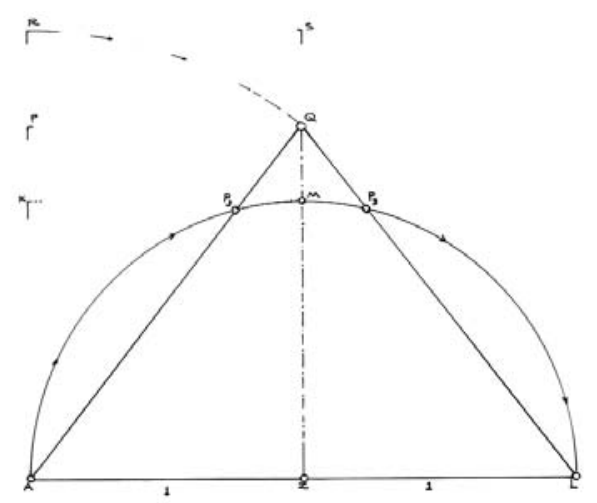

Fig. 5

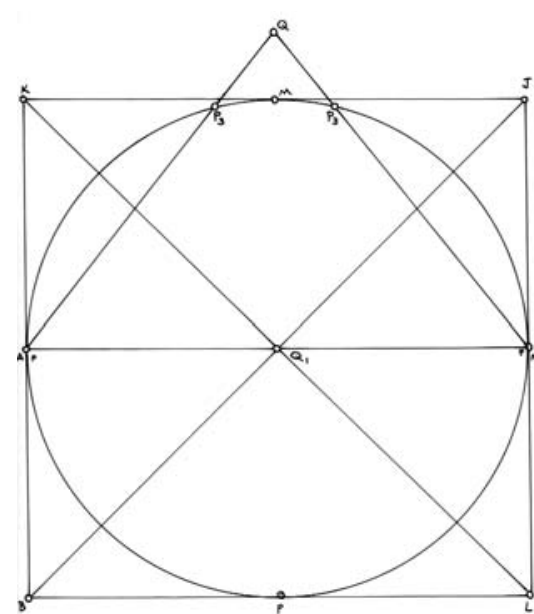

Fig.6 
the center Q1 to inscribe a circle inside the square of the base and tangent to the four sides at the midpoint $\mathrm{p}$ of the square. (In philosophical geometry, the highest shape in the hierarchy is the circle, for it represents the cosmos. On Earth, this hierarchy begins with the square. Constructing the circle in the square is one form of mandala, and can be viewed as the circle being born of the square.) The circle cuts the slopes AQ and QN of the Great Pyramid elevation at the points $\mathrm{p} 3$. It can be seen that these two points are not at the same level and height as M. The difference is subtle, almost infinitesimal, but it is there. These two points will work in tandem and harmony with the next drawing.

In Figure 7, point p3 are used to establish point p4, which is at the same height as the point p3. Point p4 will mark the radius Q1p4 of a new, smaller, concentric circle just within the first circle. This new circle will yield the size of the base of the Pyramid of the Sun, the base that is "almost" or "not quite" equal to the base of the Great Pyramid.

1. From the previous drawing, using points $\mathrm{p} 3$, draw through both $\mathrm{p} 3$ points to go through $\mathrm{p} 4$, and draw kj, a side of the base of the Pyramid of the Sun, parallel with KJ.

2. Continue this process around the other three sides. There are two ways to assist in this step.

3. Draw a second circle inside the first circle.

4. By using the two diagonals of the squares, the 45-degree lines will enable you to make the 90-degree turns to the other three sides, and to keep the second square parallel to the first.

5. Complete the second square, bkjl, parallel to BKJL.

6. This second, inner square is the base of the Pyramid of the Sun, in plan view.
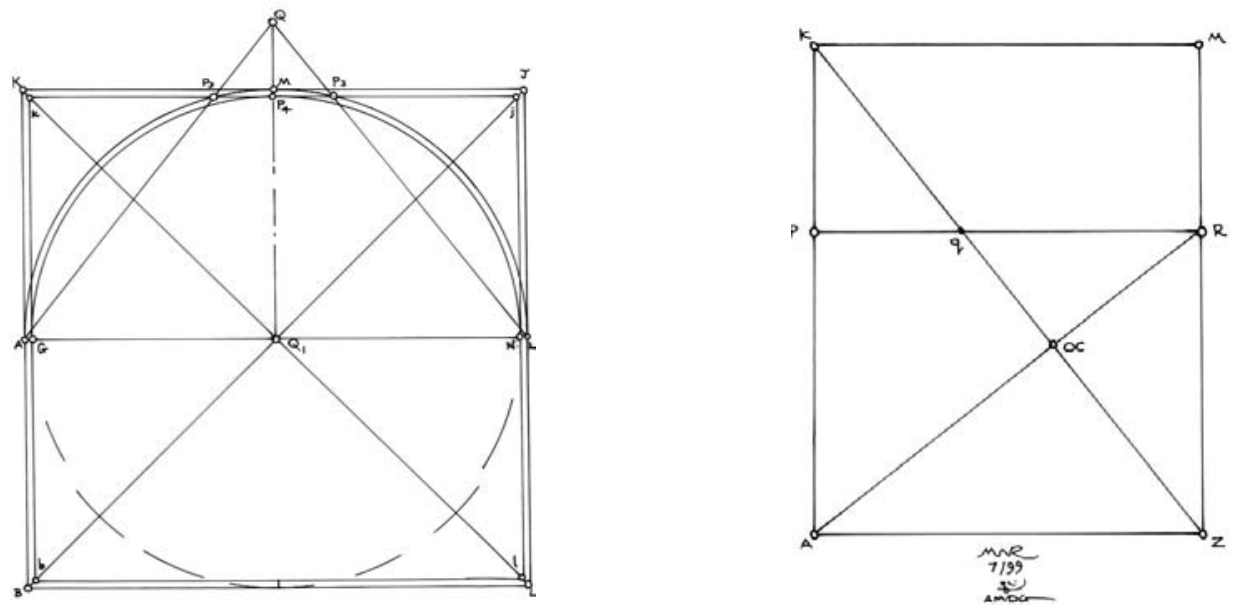

Fig.7

Fig. 8 
Now we come to the more difficult drawings, but difficult tasks bring greater rewards. The goal of the constructions is to obtain the height, the elevation of the second Pyramid. Ascertaining the results is arduous because of the destruction and abuse the structure has suffered over the centuries, most especially in the twentieth century. Unlike the Great Pyramid, which has a basically straight slope and a clearly marked capstone apex -albeit with a 9 meter high pole placed on the degraded top of the pyramid- the Sun Pyramid has courses almost like terraced steps, and its true top, possibly containing an altar space or temple, no longer exists. These slopes are at varying angles for various reasons. ${ }^{9}$

Importantly, there are two constructions that will accomplish this. They are unconnected, but both yield the same result. They are somewhat quirky, and if you were not looking for them to generate, they would both be easily missed, perhaps like the Platonic Year. ${ }^{10}$ Let us look at Figure 8:

- Rectangle $A K M Z$ is a $\sqrt{\varphi}$ rectangle.

- Rectangle APRZ is also a $\sqrt{\varphi}$ rectangle. Here, it is considered to be a reciprocal rectangle, $1 / \sqrt{ } \varphi$, as it has been constructed from the diagonal $\mathrm{KZ}$.

- $\mathrm{AR}$ is a reciprocal/diagonal. It meets the requirement of the reciprocal relationship with the diagonal of the (any) rectangle $\mathrm{KZ}$ in that it crosses the diagonal at 90 degrees. This is true at any point along the diagonal KZ.

- When this point is at OC, the result of the reciprocal being drawn from one of the

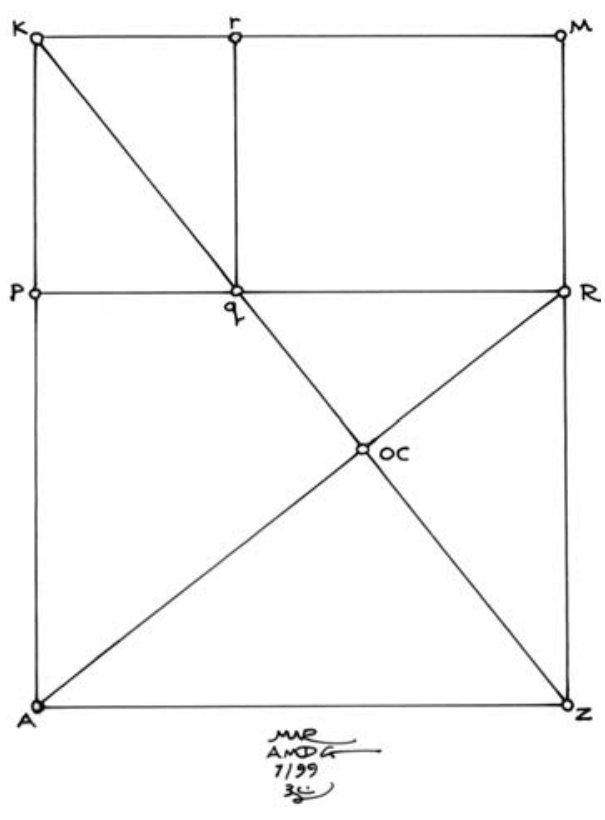

Fig.9

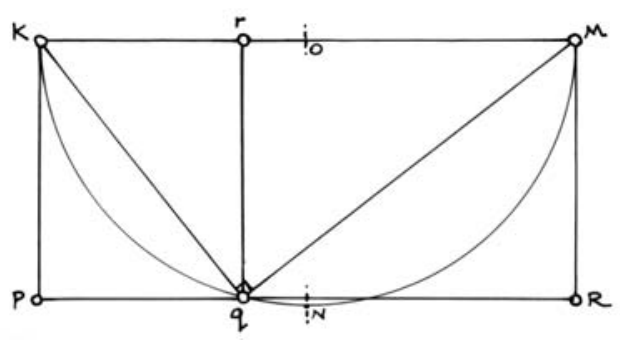

Fig. 10

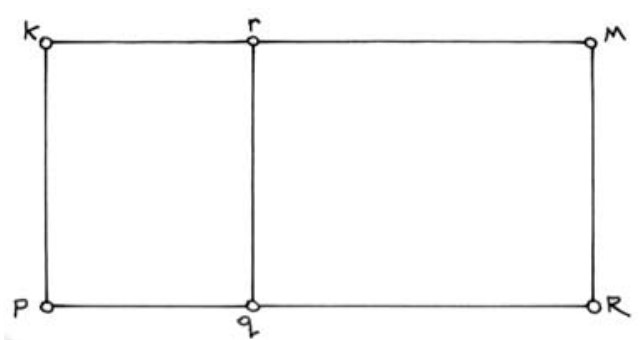

Fig. 11 
corners, here point A, OC is called an "occult center", as it is "hidden from the eye". All rectangles (except the square) have four occult centers, as there are four corners to the rectangle. These four occult centers frame the dead center of all rectangles.

- When the reciprocal is drawn as it is here, we have a diagonal/reciprocal relationship that generates a geometric progression that is based on the ratio of the rectangle in which it is drawn. Here, that ratio of the rectangle is $1: 1$. $2720196 \ldots$, so the four lengths OCR, OCZ, OCA, OCK $=1,1.272 \ldots, 1$. $618 \ldots, 2.058 \ldots$

- ONLY In the $\sqrt{\varphi}$ rectangle, the occult centers are at the golden sections of the heights and widths of the rectangle.

- Where the diagonal $\mathrm{KZ}$ cuts PR at $\mathrm{q}$ is also an occult center!

Figure 9 shows the division of the area into the reciprocal, and how it generates point $\mathrm{q}$, and that this generates three $\sqrt{ } \varphi$ rectangles (APRZ, PKrq, rqMR) within the fourth and master rectangle, AKMZ.

In Figures 10 and 11, the "2. 058..." rectangle has been isolated. In Figure 10, the Rule of Thales regarding points on the semi-circle and the 90-degree angle that is formed by drawing from that point q to the ends of the diameter KNM was applied to yield reciprocal rectangles. It can be seen here that the "2. 058..." does not contain this semi-circle, because point $\mathrm{N}$ lies outside the circumference; if it were a double square, it would contain it. The Rule of Thales

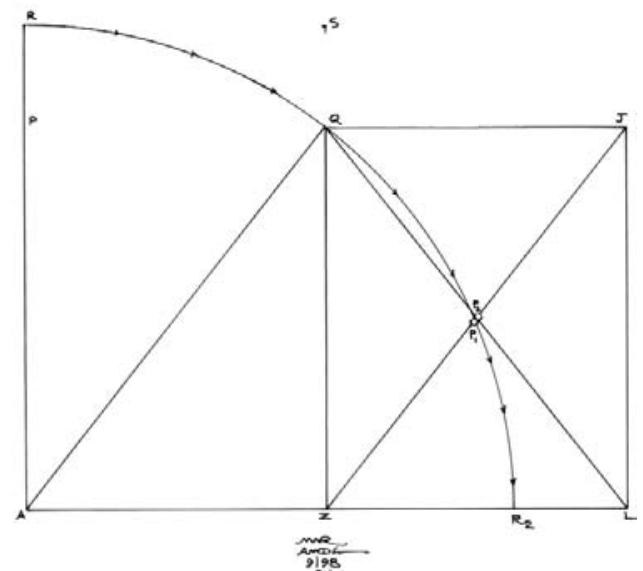

Fig. 12

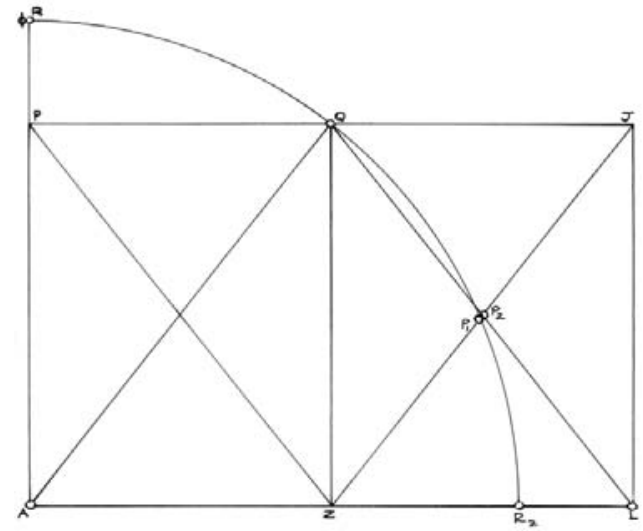

Fig. 13 
has been evoked to illustrate the function of the diagonal/reciprocal relationship, that of the crossing of the two at 90 degrees. In this instance, the angle is at the very end of diagonal qM, with $\mathrm{qK}$ as the reciprocal. In Figure 10:

- $\operatorname{PKrq}=\mathrm{qrMR}=\sqrt{ } \varphi$

- PKrq is the reciprocal rectangle to the master rectangle qrMR.

- Point $r$ is at a golden section of KM

- Point $\mathrm{q}$ is at a golden section of PR.

These construction points are esoteric in the truest sense of the word, and fall outside the realm of "normal" geometric analysis. They are not used or found in the usual procedures of construction. A rather complex grid has to be developed, and extra steps are needed to generate the specific eyes ${ }^{11}$ used in the field of the gridwork. I do, however, believe that the masons of the Pyramids knew of them, and of the unique qualities that I've outlined here. Only someone who worked or works with the ratio and the grid work for a long time would know where these "eyes" are located; only someone or some group that was incredibly close to the drawing, as I have been, would use the points that I am discussing here. These points bear no cosmic or sacred meaning for the novice, hence the esoteric nature of the constructions.

The following constructions and points are of the same nature. They are not present in the master rectangle, until a detailed and complex grid is developed. ${ }^{12}$ The two bases we have just seen are the relative sizes of the two pyramids. What an ingenious way to vary the two, with

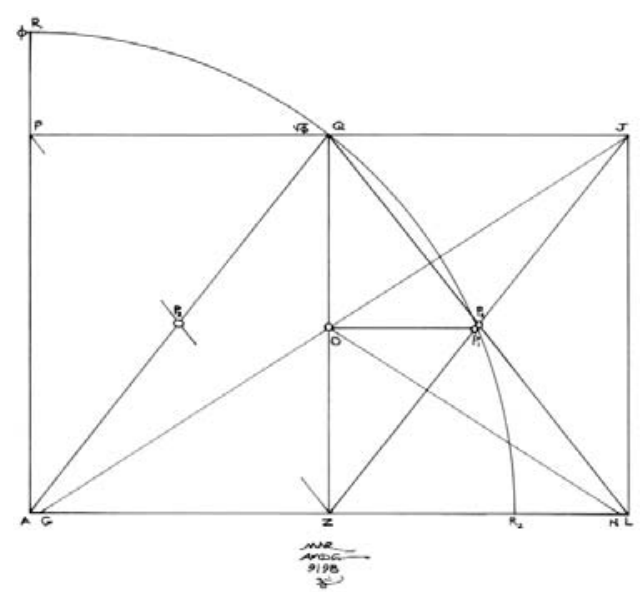

Fig. 14

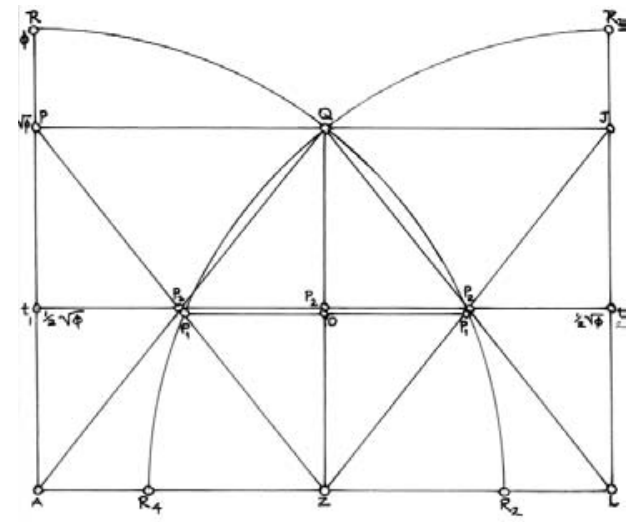

Fig. 15 
a circular curve cutting the slope.

Now we will look at the key constructions for the Pyramid of the Sun. In Figures 12 and 13, we see the elevation of the Great Pyramid, AQL. The golden section length AR is rotated through arc RR2 and stops on the base AL at R2. Two $\sqrt{ } \varphi$ rectangles are placed vertically, sharing a vertical long side ZQ and having their diagonals $\varphi$ when their short sides are 1 . The total base is now 2, with the two $\sqrt{\varphi}$ rectangles placed side-by-side. The other diagonal ZJ is drawn in AQJL. Where this arc cuts the base at R2, it divides the half-base ZL of the Great Pyramid into a golden section relationship and creates a golden section (geometric) progression across the entire base. That is:

- LR2 : R2Z :: R2Z : ZA

- $1 / \varphi: 1:: 1: \varphi$

- $\mathrm{LZ}=\mathrm{ZA}=2$

Triangle AQL is the only isosceles triangle that can do this. As it rotates, it cuts the second diagonal ZJ at p1, at the precise point needed to obtain the height of the Pyramid of the Sun. This point $\mathrm{p} 1$ is extremely close to the center of the rectangle where the two diagonals cross, point $\mathrm{p} 2$.

Next, we will construct the height of the Pyramid of the Sun from point p1 (Figure 14 and Figure 15):

1. Draw the other diagonal, $Z P$, into $\sqrt{ } \varphi$ rectangle $A P Q Z$.

2. Draw the arc JG to generate a second $\mathrm{p} 1$ in APQZ.

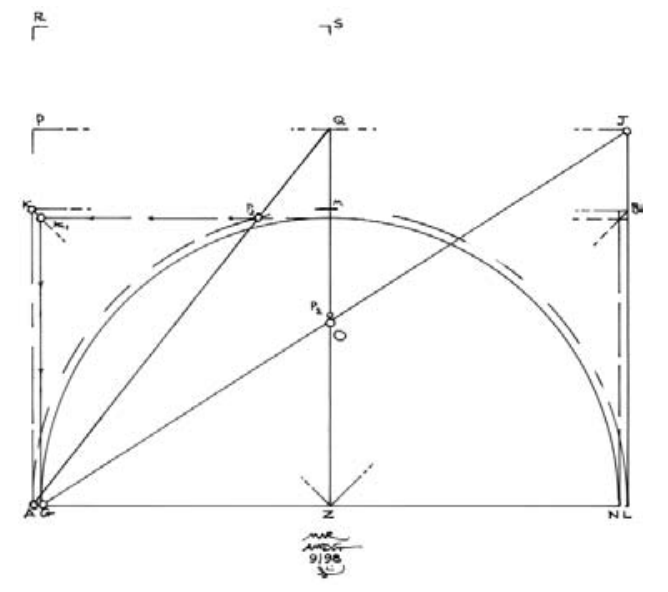

Fig. 16

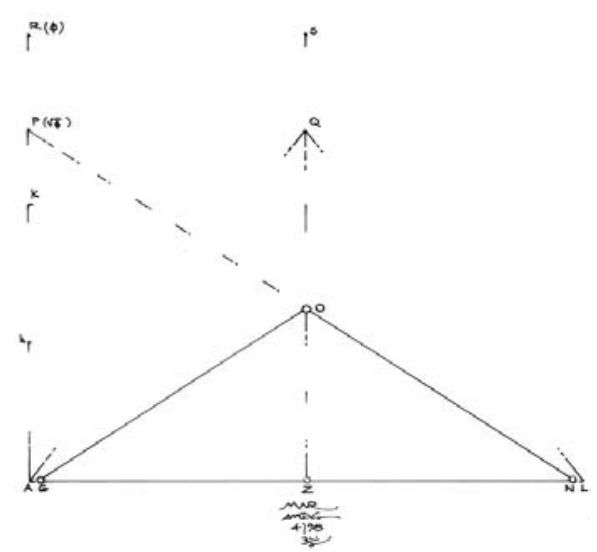

Fig. 17 
3. Draw the midlines to $\mathrm{AP}$ and $\mathrm{LJ}$ at $\mathrm{t} 1 \mathrm{p} 2$ and $\mathrm{p} 2 \mathrm{t} 2$.

4. Draw a parallel to $\mathrm{t} 1 \mathrm{t} 2$ from points $\mathrm{p} 1$ through $\mathrm{O}$.

Point $\mathrm{O}$ marks the elevation of the Pyramid of the Sun. The difference in measurement of the drawing's construction and the actual pyramids between the dead centers at $\mathrm{p} 2$ and $\mathrm{O}$ to the height ZQ in the construction is $1 / 81$. This is the very lovely and amazing decimal, $0.01234567 \ldots$ The difference between the two heights of the two pyramids is one part in approximately eighty-one and a tenth, 1 / 81.1. As a decimal, it is, $0.01233045 \ldots$ Looking at the percentage difference between my construction's measure, $1 / 81$, to the measures given by the quoted sources, which is 1 / 81.1 yields a difference of, $0.0015 \%$, an insignificant deviation.

The second method for finding the height of the Pyramid of the Sun (Figure 16) requires the same double square root of the golden section compound rectangle system used in Figure 14. In this second method, the slightly smaller base of the Pyramid of the Sun, GN, must be drawn from Figure 7. Points $\mathrm{G}$ and $\mathrm{N}$ on the base are needed.

1. Draw a line from point $G$ to point $J$, a corner of the $\sqrt{\varphi}$ rectangle on the right. (Note that if the line were drawn from point $A$, a corner of the $\sqrt{ } \varphi$ rectangle on the left, the line would cut ZQ at its mid-line. This procedure yields point $\mathrm{O}$ on $\mathrm{ZQ}$, lower on $\mathrm{ZQ}$ to the same point as was yielded in Figure 14.)

2. Repeat this procedure from point $\mathrm{N}$.

Lines GO and ON are the slope of the Pyramid of the Sun.

In each of these two methods, the height of the Pyramid of the Sun is dependent on the complete Great Pyramid construction for its measure.Next in the sequence of drawings, we complete the elevation for the Pyramid of the Sun (Figure 17). (This isosceles triangle is not intended to be the correct profile of the Pyramid of the Sun, which has stepped courses, but is instead a representation of the pyramid's height, as found by drawing through the edges of the stepped courses of the slope, and width.)

- $\mathrm{GOZ}=\mathrm{ZON}$

- GON is the elevation of the Pyramid of the Sun

If a rectangle be made with short side $\mathrm{ZO}$, long side $\mathrm{GZ}$ as the long side, and diagonal GO, the ratio of each of the two rectangles yields a ratio of $\mathrm{GZ}: \mathrm{OZ}=1: 0.6369339 \ldots$, roughly $1 / \varphi \cdot[13]$

Had the apex gone to the exact half way point (designated by the base measure GN, which is about $97 \%$ of the Great Pyramid), the rectangles would have been golden section rectangles. Although I am uncertain about the reasons for this curiosity, I do want to present it for future discussion. Perhaps this "slight" of the golden section demonstrates the builders' faithfulness to the higher plan that involves both. The builders of these structures may have been so exacting in their work that the truth may lie in a deliberate "error"! 
The Master Diagram (Figure 18) is the compilation of the individual constructions to show the overall, complete relationship between the two pyramids. Let us review its components. This construction shows the development of the golden section ratio, the progression to its square root, which yields the Triangle of Price, AQZ. The bilateral symmetry of a second Triangle of Price ZQL yields the elevation of the Great Pyramid. As the long side AR of the golden section rectangle is rotated over to its opposite side to generate the square root of $\varphi$ at $\mathrm{Q}, \mathrm{AR}$ continues down to the horizontal position at $\mathrm{R} 2$ as AR2. As the arc is swung, it cuts the diagonal ZJ of the square root rectangle ZQJL at P1. P1 yields the height of the Pyramid of the Sun at O. This height can also be generated by a diagonal line from the base vertex, $G$, to a vertex of the square root rectangle at $\mathrm{J}$, and cutting the vertical axis $\mathrm{QZ}$ at $\mathrm{O}$. The slight difference, MP4, is equal to the differences in the base perimeters. Because the chances of this coincidence occurring are quite remote, it appears that perhaps someone had done the construction originally and intentionally used this slight variation in the two pyramids. By studying the complete drawing, the beauty and elegance of the Master Construction comes through, and the connection between the two becomes much clearer.

\section{The Search for meaning: the earth, the moon and the square root of the Golden Section}

The square root of the golden section, $\sqrt{ } \varphi=1.2720195 \ldots$, is a quite remarkable number and construction. I learned of it years ago when studying spirals and the way they could be generated by various ratios, angles and lengths. ${ }^{14} \mathrm{After}$ repeated failures and several shells purchased in order
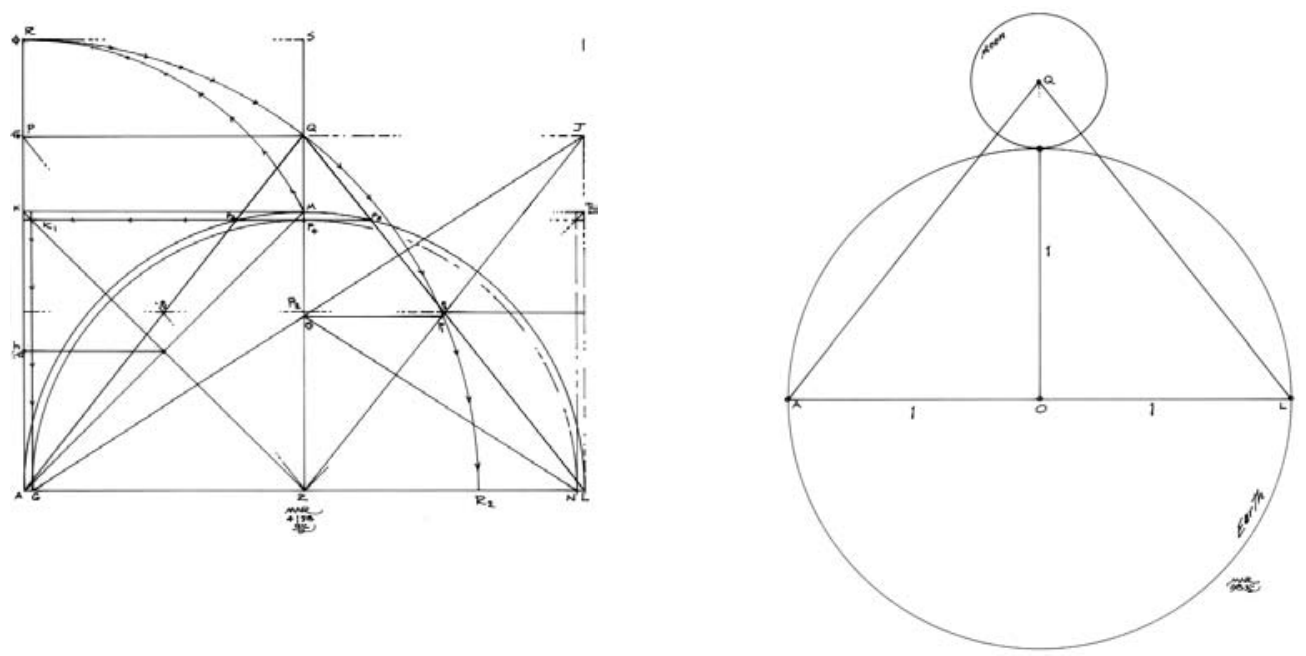

Fig. 18

Fig.19 
to substantiate the claims that the chambered nautilus was generated from the golden section rectangle, I finally realized that the correct rectangle for framing the shell is $\sqrt{ } \varphi$, not $\varphi$. I have done a great number of drawings and studies since then on the ratio, both in the rectangle and the triangle, as well as a good deal of work on the elevation of the Great Pyramid. This has brought me to a deep understanding of the number and the ratio. I believe it to be one of the basic building blocks of the natural world, and I have come to have great regard for the geometric relationships it contains and creates. It is not surprising to me that the Egyptian masters encoded this relationship into the pyramid, nor indeed that it was used for the Pyramid of the Sun.

There is also an astonishing relationship that I will mention now. Figure 19 shows the relative sizes of the Earth and our Moon. The square root of the golden section, 1.2720196... and the Great Pyramid, constructed by this number, both yield the sizes of these two heavenly orbs. This specific technique is one of the keys to understanding how the two pyramids are linked, for it is precisely where the arc from this rotation cuts the sides of the triangle AQL that yields the difference in the dimensions of the two bases we say in Figure 7.

- The moon is about $27.3 \%$ the size of the earth, or, $1.272 \ldots$ minus 1 . Figure 20 shows this relationship, as well as the squaring of the circle. Although the problem of squaring the circle was proven mathematically impossible in the 19th century (as $\pi$, being irrational, cannot be exactly measured), the Earth and the moon, the Great Pyramid and $\div \varphi$ are related to it, all coming about as close as you can get to the solution! In Figure 20:

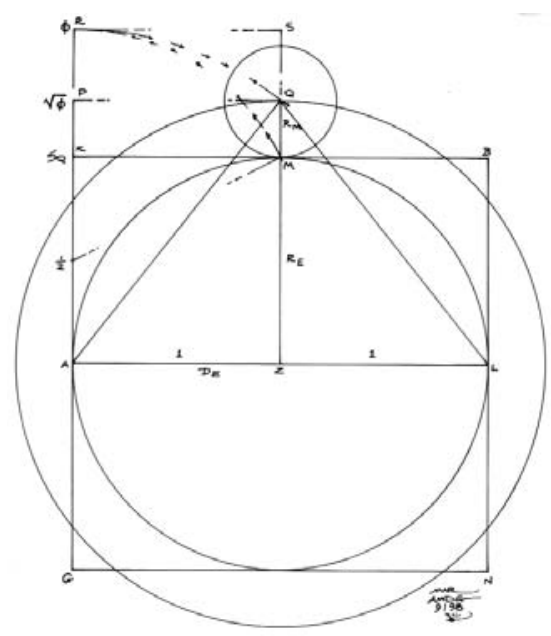

Fig. 20 
- The half-base of the Great Pyramid AZ is ONE (Unity), and is equal to the radius of the Earth.

- The base of the Great Pyramid AL is equal to 2.

- $\mathrm{L}=\mathrm{GN}=\mathrm{GK}=\mathrm{KB}=\mathrm{BN}=2$; therefore $\mathrm{GKBL}$ is a square.

- The circle inscribed within the square is the Earth.

- This circle cuts the vertical axis at M, with MQ a remainder.

- $\mathrm{Q}=\mathrm{Rm}=$ Radius of the Moon.

- With center Z, a second, concentric circle, with radius ZQ (the radius of the Earth AND the radius of the Moon added together) is circumscribed around the circle that represents the Earth.

- This new circle (radius = ZQ) and the square around the Earth, GKBN, are very nearly equal in perimeters (close enough to wonder about Nature's intentions!).

Of note is that our earth/moon relationship is the only one in our solar system that contains this unique golden section ratio that "squares the circle". Along with this is the phenomenon that the moon and the sun appear to be the same size, most clearly noticed during an eclipse. This too is true only from earth's vantage point... No other planet/moon relationship in our solar system can make this claim. If the base of the Great Pyramid is equated with the diameter of the earth, then the radius of the moon can be generated by subtracting the radius of the earth from the height of the pyramid (see Figure 16).

\section{Conclusions}

Out of all the number systems and numerical relationships that could have been used, the Egyptians selected the golden section ratio and its square root in the building of the Great Pyramid. The Pyramid of the Sun also contains a sophisticated and subtle "infusion by association" of the same ratio. Through a specific series of points within the construction of the Great Pyramid's elevation, the elevation of the Pyramid of the Sun can also be generated: the two elevations are linked through this precise geometry. That two different architects would use points in the geometric constructions in the singularly unique way I have discussed seems, to me, most improbable, perhaps impossible, for they serve neither a functional purpose, being totally unnecessary for construction purposes, nor a specific spiritual, symbolic, or philosophical purpose. However, it appears intentional. And if it was intentional, was it also intentionally mysterious? After examining the geometric analyses, it becomes evident that the two structures fit together as one geometric construction. But unless one is familiar with the various $\varphi$ and $\sqrt{ } \varphi$ constructions, this particular and unique relationship could be easily missed.

The insights afforded by geometric analysis are valuable in gaining a deeper awareness of the makers' intentions. In this particular instance, the insights led to even bigger questions. Were these two pyramids somehow related intimately in other ways than by the geometry inherent in both? Why would two pyramids be so very nearly in a 1:2 relationship in their heights, yet not "exactly" or "not at all"? After examining the constructions presented here, it seems too great a coincidence that they are "almost exactly" alike but not related.

Although the two pyramids were separated by the Atlantic Ocean, in vastly different locations and cultures, is it possible that the two pyramids were built by the same architect or 
architects, either directly, or by courier with instructions? Was the ocean transversed, or was there a continent that connected the Americas with Europe and Africa, and inhabited by intelligent and gifted people? ${ }^{15}$ However, even though the two constructions fit together in such a unique and curious way, this is not undeniable scientific or mathematic proof that the two pyramids were designed and executed by the same architects. Much more would need to be done. Documents on the subject would be grand!

As I write, it is my understanding from a number of sources that parts of the Great Pyramid were closed for a few years for the purposes of cleaning the graffiti from the walls and installing a new air conditioning and ventilation system. In addition, rumors persist that ancient texts exist at the Gizeh complex. Modern underground sonar studies indicate that there is a large room or space under the paws and belly of the Great Sphinx, and that there is a second "gallery" that extends from the Sphinx to a large hall or room directly under the Pyramid along an east/west axis. The rumor advanced with these stories is that, under the cover of the edifice itself, away from public view, workers have begun the descent inside, straight down from the apex of the pyramid; at the specific depth indicated by a scan of the area already undertaken, they will turn due east toward the Sphinx . Their intent is to reach this corridor and to follow it to the area recorded on the sonar. The echoes indicate that there is a hollow area under the Sphinx, and officials within the government have remained tightlipped and have refused further study and investigation to be conducted by anyone outside their auspices. The commonly held belief is that this hall is the fabled Hall of Records, and contains writings and documents that explain the origins and history of mankind on the planet. Perhaps there are documents that link these two pyramids!

Although there can be no final declaration for or against the hypothesis I have presented about these two pyramids being linked by a common builder or building culture, I hope that I have at least opened the possibility for further work on the subject. Although ragged and worn, each building stands as a memorial to something that was marvelous, and is still mysterious. They serve to remind us also that the human body, mind and spirit have no boundaries.

\section{Notes}

1. The Great Pyramid is so massive that a plumbline will not hang straight down when near the pyramid but will swing toward the structure. Cf. Tompkins, Secrets of the Great Pyramids, pp. 84-85, where Tompkins, discussing the measurements taken by Piazzi Smyth, writes "To obtain the correct latitude of the Great Pyramid without having his plumb line diverted from the perpendicular (italics are mine) by the attraction of the huge bulk of the Pyramid, Smyth made his observations from the very summit; there the Pyramid's pull of gravity would be directly downward."

2. One study expressing such scepticism is George Markovsky, "Misconceptions about the Golden Ratio", The College Mathematics Journal, 23, 1, (January 1992), pp. 2-19.

3. As most of us are aware, there are volumes of writings and studies that address the values of $\pi$, but for those who are not acquainted with the literature, I recommend Petr Beckmann's A History of Pi, and any one of the books written by John Michell on the subject of ancient measures, especially The New View Over Atlantis and The Dimensions of Paradise. Michael Schneider was kind enough recently to draw my attention to another fraction used in antiquity: 355/113. Also, as John Ivimy points out in The Sphinx and the Megaliths, Chap. 9, p. 116, A. Thom's studies of ancient and Megalithic sites indicate that the geometers of the era abhorred incommensurables. I am in total agreement with this view. As I have already mentioned, the focus needs necessarily to be on the intentions of the builders, and even on the cleverness and intuitive powers of the ancient mind to solve an irrational problem with whole numbers and fractions contrived from natural integers. Most importantly, it has been shown by a 
number of writers and scholars (including R. A. Schwaller de Lubicz, Robert Lawlor, and Peter Tompkins) that what is more significant is not in the accuracy of $\pi$, but rather, its connection with the square root of the golden section, $\sqrt{ } \varphi$, in the formula: $4 / \pi=\sqrt{ } \varphi$. This equation leads to the problem of quadrature, the squaring of the circle.

4. For a thorough text on the measures of the Great Pyramid, cf. the Appendix written by Livio Catullo Stecchini in Tompkins, Secrets of the Great Pyramid. I also recommend Tompkins' history of the great tradition of measures that have been done on the Great Pyramid and the Gizeh Complex, and the measurers/ geometers who did them.

5. Cf. Schwaller, The Temple of Man, Chapter 5, "Foundations of Pharaonic Mathematics", pp.88-125.

6. Cf. Euclid, The Elements, Book vi, definition 3.

7. Cf. Euclid, Book xiii, proposition 8.

8. Robert Lawlor addresses the symbolism of these generative powers of $\varphi$ in Sacred Geometry, pp. 44-64.

9. For excellent reading and for references into the pyramids of the Teotihuacan Complex, cf. Tompkins, Mysteries of the Mexican Pyramids, Chapter 18, "Mathematical Extrapolations", pp.241-263. Peter Tompkins does an excellent job of weeding and clearing up Hugh Harleson's extrapolations. I don't agree with his measuring schema that he calls the hunab, a special measure remotely similar to Thom's megalithic yard.

10. I've been working with the $\sqrt{\varphi}$ ratio for a long time and I know these peculiar items well. By example, I will define one of the quirky things about the $\sqrt{ } \varphi$ function. Multiplying $\sqrt{ } \varphi$ and $\varphi$, we get $1.2720196 \ldots \times 1.6180339 \ldots=2$. $058170 \ldots$, an amazing and curious number. By subtracting Unity from it, you will have the number, 1. 058170... The frequency ratio for the 12 equal parts, or notes, of even- tempered tuning for musical instruments is: 1 . 059463... Simply stated, a string will vibrate when plucked, and will sound some note. Then, if the string is shortened or lengthened to the next note up or down, it will carry the original note with it to the next note, by the note and dividing (lower) or multiplying (higher) by the constant, 1. 059463 ...(Cf. Trudi Hammel Garland, Math and Music, pp. 40-43). The deviation, minus the One, is - $0.114229 \ldots \%$ low. What is the significance of subtracting Unity? The cutting off of squares, as Hambidge points out in Dynamic Symmetry, was a design practice of the Greeks. It is also used by the Greek mathematicians with whom the artists and designers collaborated, to study "compound ratios" that were generated from the addition and subtraction of the square, One. Following then, if a rectangle be drawn that has a short side equal to one, and the long side equal to $\sqrt{ } \varphi \times \varphi$, or $05817 \ldots$ and a square is cut off on the long side, the remaining rectangle will be only about . $001 \ldots$ from this frequency ratio. Now the rectangle will not necessarily make music, but the geometer/artist or builder believes that by infusing these types of number concepts into geometric shapes that the geometry will have a certain quality, a function, to a higher order. The second part of this example is a more subtle and technical one in that when the "2. 058..." rectangle is drawn out, or is generated by the harmonic deconstruction of the $\sqrt{ } \varphi$ rectangle (see Illustration 1 above), this rectangle is created when the reciprocal is drawn. The remainder is this rectangle. The first instinctual response is to think that it is a $\sqrt{ } 4$ rectangle, the double square, 2 to 1 . There is only a $.058 \ldots$ difference between the two.

11. An "eye" is where two or more lines intersect. These points are valuable in the generation of more complex geometric relationships from simple initial constructions. The more they are used, the more they create new eyes, and they are quite similar to fractals. In traditional geometry, where these diagonals cross in the middle is known as the arithmetic center, rather than a geometric one, because it is one half the distance from all four corners, and it demonstrates simple equality. This particular eye $\mathrm{p} 1$ is not one that would ordinarily be considered or used in a design. It would be far more logical to simply use the arithmetic mean, half way, of the height, if the builders are making references to twice or four times $\mathrm{p}$ in the relationship of base perimeter to height. Rather, they used this second and far more hidden point, p1, to build with. The masters who built the pyramids were precise, and I find it is unlikely that this "slightly less than half the height" was accidental.

12. Additionally, in the following drawings, it should be kept in mind that we are performing the function of multiplicity and regeneration by placing two $\sqrt{ } \varphi$ rectangles side-by-side on the $1.272 \ldots$ sides. Their bases, as the Great Pyramid itself, have the value of two, not one.

13. The deviation is, curiously, 3. 058... high (like our $\varphi \times \sqrt{ } \varphi$ number, $2.058 \ldots$ but this time with Unity added, not subtracted!); see note 10 .

14. The Curves of Life by Theodore A. Cook is far and away the greatest work on the subject.

15. Both Plato's "Timaeus" and "Critias" speak with certitude that the continent of Atlantis did exist. John Michell, the great British scholar and author of dozens of books on ancient and universal canons of measure, in The Dimensions of Paradise, even goes into the dimensions of the fabled land, taking Plato at his word. 


\section{References}

Petr Beckmann. A History of $\pi$. New York: The Golem Press, 1971.

Walus E.A. Budge. The Egyptian Heaven and Hell. Chicago: Open Court Publishing Co., 1925.

Theodore Andrea Cook. The Curves of Life. New York: Dover, 1979.

EuCLID. The Thirteen Books on the Elements. Thomas L. Heath, trans. New York: Dover, 1956.

Alain Erlande-Brandenburg. Cathedrals and Castles: Building in the Middle Ages. New York: Harry N. Abrams, 1995.

Marco Frascari and Livio Volpi Ghirardini. "Contra Divinam Proportionem." Nexus II: Architecture and Mathematics. Kim Williams, ed. Fucecchio,Florence: Edizioni dell’ Erba, 1998.

Hugh Harleson. "A Mathematical Analysis of Teotihuacan."Mexico City, XLI International Congress of Americanists, October 3, 1974.

Trudi Hammel Garland. Math and Music. Palo Alto: Dale Seymour, 1995.

Manly P. Hall. The Secret Teachings of All Ages. Los Angeles: The Philosophical Research Society, 1988.

Jay Hambidge. The Elements of Dynamic Symmetry. New York: Dover, 1967.

Graham Hancock. Fingerprints of the Gods. New York: Crown Trade Paperbacks, 1995.

John Ivimy. The Sphinx and the Megaliths. London: Abacus, 1976.

RoberT LaWlor. Sacred Geometry. London: Thames and Hudson, 1982.

A.T. ManN. Sacred Architecture. Rockport: Element Books, 1993.

George Markowsky. "Misconceptions about the Golden Ratio." The College Mathematics Journal, vol. 23, no. 1 (January 1992), pp. 2-19.

ERnest G. MCClain. The Myth of Invariance. York Beach: Nicolas-Hays, 1976.

JoHn Michell. The Dimensions of Paradise. London: Thames \& Hudson, 1988.

JoHn MichelL. New Light on the Ancient Mystery of Glastonbury. London: Gothic Image, 1990.

John Michell. New View Over Atlantis. London: Thames \& Hudson, 1983.

PLATO. Timaeus and Critias. Desmond Lee, trans. London: Penguin Books, 1965.

Michael SCHneider. The Beginners Guide to Constructing the Universe. New York: Harper Collins, 1995.

René A. SChWAller De LubicZ. The Egyptian Miracle. Rochester: Inner Traditions International, 1988.

René A. SChWAller De Lubicz. Sacred Science. Rochester: Inner Traditions International, 1988.

René A. SCHWALler de Lubicz. The Temple of Man. 2 volumes. Rochester: Inner Traditions International, 1998.

D’Arcy Wentworth Thompson. On Growth and Form. New York: Dover, 1992.

Peter Tompkins. Mysteries of the Mexican Pyramids. New York: Harper \& Row, 1976.

Peter Tompkins. Secrets of the Great Pyramid. New York: Harper Collins, 1971.

\section{About the author}

Mark Reynolds is a visual artist who works primarily in drawing, printmaking and mixed media. He received his Bachelor's and Master's Degrees in Art and Art Education at Towson University in Maryland. He was awarded the Andelot Fellowship to do post-graduate work in drawing and printmaking at the University of Delaware. He is also an educator who teaches sacred geometry, linear perspective, drawing and printmaking to both graduate and undergraduate students at the Academy of Art College in San Francisco, California. He was voted Outstanding Educator of the Year by the students in 1992. Additionally, he is a geometer, and his specialties in this field include doing geometric analyses of architecture, paintings and design. He has just completed measurements of the Pazzi Chapel in Florence in order to do a comprehensive analysis of the geometry present in the chapel, to be published in Nexus III: Architecture and Mathematics (Pisa: Pacini Editori, 2000) and for a presentation he will be giving at the Nexus 2000 Conference, Ferrara, Italy, 4-7 June 2000. For the past decade, Mr. Reynolds has been at work on an extensive body of drawings, paintings and prints that incorporate and explore the ancient science of sacred, or contemplative, geometry. He is widely exhibited, showing his work in group competitions and one person shows, especially in California. His work is in corporate, public, and private collections, and he is represented by the Mill \& Short Gallery in San Francisco. He is a member of the California Society of Printmakers, the Los Angeles Printmaking Society and the Marin Arts Council. He currently lives with his wife and family in Mill Valley, California. 